\title{
Temporal and spatial determination of EEG-seizure onset in the frequency domain
}

\author{
O. Blanke ${ }^{a, c}$, G. Lantz ${ }^{\text {b, c }}$, M. Seeck ${ }^{a}$, L. Spinelli ${ }^{\text {a, c }}$, R. Grave de Peralta ${ }^{c}$, G. Thut $^{\text {b, c }}$, \\ T. Landis ${ }^{\mathrm{c}}$, C.M. Michel ${ }^{\mathrm{b}, \mathrm{c}, *}$ \\ ${ }^{a}$ Laboratory of Presurgical Epilepsy Evaluation, Department of Neurology, University Hospital, 24 rue Micheli-du-Crest, 1211 Geneva, Switzerland \\ ${ }^{\mathrm{b}}$ Plurifaculty Program of Cognitive Neurosciences, Department of Neurology, University Hospital, 24 rue Micheli-du-Crest, 1211 Geneva, Switzerland \\ ${ }^{\mathrm{c}}$ Human Brain Mapping Laboratory, Department of Neurology, University Hospital, 24 rue Micheli-du-Crest, 1211 Geneva, Switzerland
}

Accepted 21 December 1999

\begin{abstract}
Objective: A quantitative analysis of scalp electric fields in patients suffering from pharmacoresistant temporal lobe epilepsy was performed in order to study the development of rhythmic ictal activities over time.

Methods: A method that calculates phase-corrected voltage maps in the frequency domain (FFT-approximation) was applied to ictal multichannel recordings in 10 epileptic patients. The onset of the ictally dominant frequency was determined and its temporal evolution over a time period of $46 \mathrm{~s}$ around the ictal EEG onset was studied. The analysis was completed by a linear inverse solution that estimated the sources of the dominant frequency.

Results: This method permitted the identification of an ictally dominant frequency which started on the average prior to the onset of initial EEG signs as determined by visual inspection. The frequency incremented during the evolution of the seizure in all patients. The linear inverse solution algorithm localized the source of this frequency to the brain region which was clinically determined as the site of seizure onset and whose resection rendered all patients seizure-free.

Conclusions: Our data suggest that the constant increase of the ictally dominant frequency is related to the amount of temporal lobe tissue generating the ictal discharges. Frequential analysis of ictal electric fields can be reliably used to detect focal pathological activity early during seizure onset arising in deep structures such as the mesial temporal lobe. (C) 2000 Elsevier Science Ireland Ltd. All rights reserved.
\end{abstract}

Keywords: Temporal lobe epilepsy; EEG; Scalp electric fields; Frequency domain; Seizure propagation

\section{Introduction}

Many patients with medically refractory complex partial seizures of temporal lobe origin can benefit from neurosurgical treatment. The non-invasive presurgical evaluation process usually includes the recording of multiple seizures by means of video-EEG from scalp and sphenoidal electrodes in order to localize the epileptogenic region. This region is defined as the smallest area of the brain whose removal results in a total cessation of seizures (Engel et al., 1993).

Different spectral changes of the EEG have been described in patients with complex partial seizures (Geiger and Harner, 1978; Blume et al., 1984). The most frequent pattern is characterized by a widespread attenuation of background activity, without topographic value, followed

* Corresponding author. Tel.: +41-22-372-8323; fax: +41-22-3728340 .

E-mail address: christoph.michel@hcuge.ch (C.M. Michel) by the appearance of rhythmic frequencies containing localizing information (Quesney et al., 1993). When analyzing these lateralized rhythmic patterns, Risinger et al. (1989) found a high correlation between ictal patterns of $5 \mathrm{~Hz}$ or faster frequencies in a sphenoidal and/or temporal location and the presence of an ipsilateral mesial temporal seizure onset. Although this pattern was quite specific, it unfortunately had a poor sensitivity of only $40-50 \%$. The latter finding was confirmed by Ebersole and Pacia (1996) who proposed a frequential differentiation between seizures arising in the lateral or the mesial temporal cortex. They found that seizures originating in the lateral temporal lobe can be characterized by a typical frequency pattern of 2-5 Hz. They also noted a low stability and commonly observed morphological and frequential changes early during the seizure for this ictal pattern (Ebersole and Pacia, 1996). However, there has been some discussion about whether and when seizures originating in the mesial temporal lobe can be detected on the surface EEG. Studies which analyzed 
seizure propagation in depth and surface recordings in temporal lobe epilepsy have suggested that visual EEGanalysis can only detect ictal activity once it has spread from mesial to neocortical structures (Spencer et al., 1990; Pacia and Ebersole, 1997). Thus it is a drawback for the presurgical work-up that the visual EEG patterns which provide the localizing and lateralizing information about the seizure origin are not a 'direct manifestation of cerebral activity at seizure onset' (Pacia and Ebersole, 1997) and only result from current spread during later ictal evolution. The combination of quantitative methods and multichannel EEG recordings might help to detect frequential changes early and prior to the visually estimated seizure onset. Recent investigations have shown that the spectral properties during the phase which follows rhythmic seizure onset can be retrieved (Gotman et al., 1993; Alcaron et al., 1995). By approximating phase corrected voltage maps in the frequency domain, Lantz et al. (1999) have calculated the full scalp spectrum for ictally dominant frequencies, by using the so-called FFT Approximation method (Lehmann and Michel, 1990) and subsequently used these voltage maps to localize the mesiotemporal epileptic focus. Moreover, a recent preliminary report from our group suggests that the calculation of the full scalp spectrum in the frequency domain can detect pathological ictal rhythms earlier in time than revealed by pure visual inspection of the EEG (Michel et al., 1999).

However, the time of onset as well as the temporal evolution of these ictal spectral changes and their relation to electroencephalographic signs - as revealed by visual EEG analysis - have been investigated systematically. The aim of the present study was to investigate the onset and temporal evolution of ictally dominant frequencies in a group of patients with a highly probable seizure onset in the mesiotemporal lobe. We completed our analysis by using a linear inverse solution in order to localize the sources of these frequencies and compare them with the brain region whose resection rendered all patients seizure-free. Preliminary results have been published previously (Blanke et al., 1997).

\section{Methods}

\subsection{Patients}

Cases were selected of candidates for partial temporal lobectomies (TLE) with medically refractory epilepsy. The operations were performed at the University Hospital of Geneva, Switzerland, or at the Lund University Hospital, Sweden. All patients had a similar evaluation that included interictal and ictal video-EEG, neuropsychological testing, interictal and ictal SPECT (single photon emission computer tomography), brain magnetic resonance imaging (high resolution in seven cases), in seven cases interictal PET (positron emission tomography) and neuropathological analysis of the resected tissue. Patients fulfilling the following two criteria were included in the analysis: (1) patients with maximal ictal activity in the sphenoidal electrode and/ or temporal electrode with clear focal rhythmic activity (Risinger et al., 1989); (2) patients evaluated with highresolution MRI $(n=7)$ who had signs of unilateral hippocampal sclerosis on MRI scans (diminished volume of the hippocampus and/or T2-signal enhancement) or patients

Table 1

Clinical information on all patients ${ }^{\mathrm{a}}$

\begin{tabular}{|c|c|c|c|c|c|c|}
\hline Patient & Age & Ictal EEG & MRI & PET & SPECT & Pathology \\
\hline 1 & 44 & Sp1, F7, T3 & Normal* & - & Left fronto-temporal & - \\
\hline 2 & 28 & Sp1, F7, T3 & Normal* & - & Left fronto-temporal & Unspecific \\
\hline 3 & 41 & Sp1, F7, T3 & Wide left temporal horn* & - & $\begin{array}{l}\text { Bitemporal } \\
\text { (Iomazenil: left } \\
\text { temporal) }\end{array}$ & Left hippocampal sclerosis \\
\hline 4 & 20 & Sp1, FT7, F7 & Left hippocampal sclerosis & Left temporal & Left temporal & Left hippocampal sclerosis \\
\hline 5 & 33 & Sp1, FT7, T3 & $\begin{array}{l}\text { Left hippocampal sclerosis, } \\
\text { left anterior temporal } \\
\text { atrophy }\end{array}$ & Left temporal & Left temporal & $\begin{array}{l}\text { Left hippocampal sclerosis, } \\
\text { discrete temporal } \\
\text { neocortical gliosis }\end{array}$ \\
\hline 6 & 44 & F7, T3, Sp1 & $\begin{array}{l}\text { Left hippocampal sclerosis, } \\
\text { discrete atrophy of the left } \\
\text { hemisphere }\end{array}$ & Left temporal & Left temporal & Unspecific \\
\hline 7 & 31 & Sp1, T3, F7 & Left hippocampal sclerosis & Left temporal & Left temporal & Left hippocampal sclerosis \\
\hline 8 & 46 & $\mathrm{Sp} 2, \mathrm{~T} 4, \mathrm{~F} 8$ & Right hippocampal sclerosis & Right temporal & Right temporal & $\begin{array}{l}\text { Right hippocampal sclerosis, } \\
\text { discrete temporal } \\
\text { neocortical gliosis }\end{array}$ \\
\hline 9 & 19 & Sp2, F8, AF8 & Right hippocampal sclerosis & Normal & Left hemispheric & Unspecific \\
\hline 10 & 21 & Sp2, F8, T4 & Right hippocampal sclerosis & Right temporal & Right temporal & Right hippocampal sclerosis \\
\hline
\end{tabular}

${ }^{a}$ An asterisk (*) in the MRI column indicates that low-resolution MRI has been carried out. In patient 9 a discrepancy can be noted between SPECT findings (left hemispheric) and other results from the presurgical workup (right temporal). However the patient was included in the study since inclusion criteria were based on EEG and MRI findings. Note that patient 9 as well as all the other patients have been seizure-free for more than 24 months following the operation. 
who were investigated with low resolution MRI $(n=3)$ and had a normal MRI. Ten patients were included in the study. Clinical findings are depicted in Table 1. Left partial anterior temporal lobectomy was carried out in seven patients and right partial temporal lobectomy in the three remaining cases. Neuropathological examination of the resected tissue confirmed hippocampal sclerosis in 6 of 9 patients, 3 patients showed unspecific changes, one patient was not investigated (Table 1). After the operation, all patients had excellent outcome (seizure free for $>24$ months), consistent with class 1 of the Engel classification (Engel, 1993).

\subsection{Visual video-EEG analysis}

Seizures were recorded with 28 or 31 electrodes, placed according to the 10-20 system, with special coverage of the temporal lobes and bilateral sphenoidal wire electrodes (Deltamed equipment). In each patient three clinically and electroencephalographically representative seizures were selected. We only included complex partial seizures with continuous rhythmic EEG changes. For all seizures, the electroencephalographic seizure onset (Fig. 1; ESO = focal or non-focal sharp- and slow waves, spikes or spike wave complexes) and the onset of clear continuous focal rhythmic activity (Fig. 1; RSO = well modulated rhythms of temporal location (Risinger et al., 1989; Ebersole and Pacia, 1996) were determined by visual video-EEG analysis. After excluding the signals recorded with the sphenoidal electrodes, the data were further analyzed using the following methodological approach.

\subsection{Identification of the ictally dominant frequency band and determination of its temporal evolution}

The time period chosen for the analysis was from $32 \mathrm{~s}$ before to $14 \mathrm{~s}$ after the ESO. We did not include the onset of ictal behavior to select the ictal time period, since it has been shown in previous studies that ictal EEG analysis alone can reliably define the seizure onset (Risinger et al., 1989; Ebersole and Pacia, 1996; Lantz et al., 1999). The selected 46 s time period was divided into 23 non-overlapping 2 s epochs. The $2 \mathrm{~s}$ epochs were collected consecutively, without the exclusion of epochs containing eye movements or other artifacts. Each epoch was transformed into the frequency domain with a resolution of $0.5 \mathrm{~Hz}$ by the FFT-Approximation which has been described previously (Lehmann and

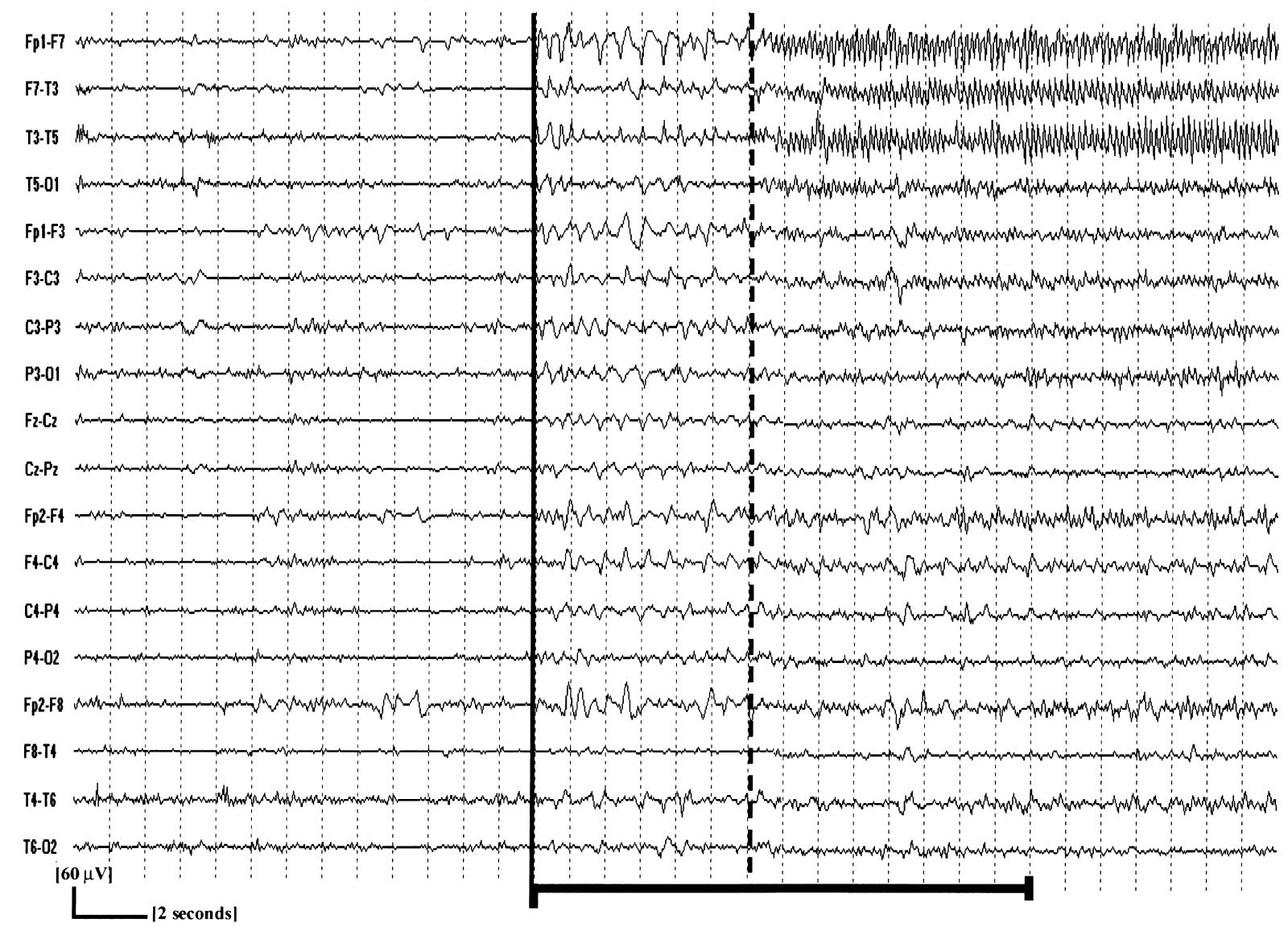

Fig. 1. Typical seizure with temporal onset. Seizure onset recorded with 31 electrodes shown in a bipolar montage. Electrode names are shown on the left. A period of $34 \mathrm{~s}$ around the electroencephalographic seizure onset (vertical black line) is shown. Dashed line indicates rhythmic seizure onset and the horizontal black line indicates the seven consecutive $2 \mathrm{~s}$ time epochs which were chosen to determine the ictally dominant frequencies for each seizure. EEG recording shows left temporal seizure onset. 
Michel, 1990). This reference-independent technique incorporates the phase angles between the different electrodes in order to derive a non-ambiguous statement about the electric field distribution for each frequency point. The FFT-Approximation method provides - in contrary to the analysis of power maps - voltage maps with positive and negative values (as the raw EEG-data). This method as been applied in different data sets (Michel et al., 1992, 1995; Dierks et al., 1993; Lehmann et al., 1993) including EEG of epileptic patients (Lantz et al., 1999; Michel et al., 1999). Fore each seizure in each patient, the 7 FFT-approximated epochs following the ESO were averaged. From this average prominent power peaks in the frequency spectrum were identified. Frequencies below $2.5 \mathrm{~Hz}$ were considered to be mainly due to eye artifacts, and were therefore excluded from the analysis. The frequency with maximal global field power (GFP, Michel et al., 1995) which were consistent across the three evaluated seizures were identified in each patient and subsequently analyzed. The range of consistency was defined as the frequencies within $\pm 0.5 \mathrm{~Hz}$ around the prominent power peak in the frequency spectrum of each seizure (see Fig. 2). The GFP of these dominant and consistent frequencies was then determined for each of the 23 consecutive 2 s epochs (E), thus giving the temporal evolution of the strength of the electric field in the particular frequency (Fig. 3). The mean value of the temporal evolution of the dominant frequency for the three seizures was calculated per patient. The time period was determined during which the dominant frequency had maximal strength (dominant epoch, DE). By following the dominant and consistent frequencies back in time from the DE, the point in time when the band power started to rise could be determined. This point in time was considered as the seizure onset.

\subsection{Three-dimensional localization of seizure onset}

In order to estimate the localization of the ictal spectrum, we applied a 3-dimensional source localization method for the dominant and consistent frequency. For this purpose the $2 \mathrm{~s}$ epoch showing the highest GFP during the evaluated period was selected (DE). The EEG of all patients was interpolated to the same standard 21 electrode setup. This procedure was carried out, because special coverage of certain brain areas (as used over the temporal lobes during the presurgical evaluation in our patients) leads to a nonuniform coverage of the whole scalp and can disrupt proper source localization (Spinelli et al., 1997). Finally, the surface maps corresponding to the dominant and consistent frequencies were used to localize the source of each frequency map using a linear inverse solution algorithm: Radially Weighted Minimum Norm (Grave de Peralta and Gonzalez, 1998; Michel et al., 1999). The use of the linear inverse solution will allow to control that the analyzed frequency is generated approximately within the brain region whose resection rendered all patients seizure-free. We choose the Radially Weighted Minimum Norm solution because it avoids the undesirable tendency of the Minimum Norm solution to reach maxima only at the borders of the solution space while reflecting (from a physical point of view) the boundary character of the current density vector. Besides its simplicity this solution has shown a performance comparable or better than more complex solutions in the analysis of epileptic data and evoked potentials (Grave de Peralta, 1999; Michel et al., 1999). The solutions were calculated on a regular grid of 1152 nodes, using a threeshell spherical model.

\section{Results}

\subsection{Ictal frequency bands and their temporal evolution}

In all patients an ictally dominant and consistent frequency of $>5 \mathrm{~Hz}$ could be determined, which has been found in previous studies to be predictive of mesiotemporal seizure onset (Fig. 2, Table 2; Risinger et al., 1989; Ebersole and Pacia, 1996; Lantz et al., 1999). Over all patients the mean of this frequency was determined at $7.0 \pm 0.8 \mathrm{~Hz}$ ). However in 6 of 10 patients a second dominant frequency peak between 3.0 and $5.5 \mathrm{~Hz}$ was found (mean: $4.0 \pm 1.0$ $\mathrm{Hz}$; Table 2, Fig. 2). The ictally dominant frequencies in three seizures per patient ranged thus from 3.0 to $8.5 \mathrm{~Hz}$ (Fig. 2a-j). In patient 1 two dominant frequencies were determined for all three seizures with one peak at 4.0-4.5 $\mathrm{Hz}$ (mean $4.5 \mathrm{~Hz}$ ) and a second one at 7.0-7.5 Hz (mean $7.5 \mathrm{~Hz}$ ). The gray area in Fig. 2a represents the ictally dominant frequency band which includes the peak frequency of each single seizures $\pm 0.5 \mathrm{~Hz}$. The initial rise of the lower frequency was determined in the fourth $2 \mathrm{~s}$ time epoch prior to the ESO (E-4) and the DE was found in the second $2 \mathrm{~s}$ time epoch following to the ESO (E-2). The initial increase for the $7.5 \mathrm{~Hz}$ frequency in this patient was detected in the third $2 \mathrm{~s}$ time epoch prior to the ESO (E-2, see Fig. 3a) an was maximal during E7. Patient 2 showed very similar results concerning the dominant frequencies and their temporal evolution. The dominant mean frequencies were found at 3.5 and $7.0 \mathrm{~Hz}$ (Fig. 2b) and again the initial rise and the DE of the lower frequency (initial rise: E-5; DE: E1) was preceding the respective epochs of the higher frequency (Fig. 3b, initial rise: E1; DE: E5). Two dominant frequencies were also found in patients 6, 7, 9 and 10 (Fig. 2). In patients 7 (Fig. 3g) and 9 (Fig. 3i) the higher mean frequency had its DE prior to the peak of the lower frequency band. In patient 7 the lower mean frequency was at $4.5 \mathrm{~Hz}(7.5 \mathrm{~Hz})$ with an initial rise at E-2 (E-2) and the DE at E6 (E2, values in brackets give values for $7.5 \mathrm{~Hz}$ frequency). In patient 9 the lower mean frequency was at $5.5 \mathrm{~Hz}(8.5 \mathrm{~Hz})$ with an initial rise at E-1 (E-2) and the DE at E6 (E3). In patients 6 (Fig. 3f) and 10 (Fig. 3j) both frequencies showed a similar temporal evolution. In patient 10 both dominant frequencies (3.0 and 6.0 $\mathrm{Hz}$ ) showed their initial rise during E-1 and their DE during 
a)

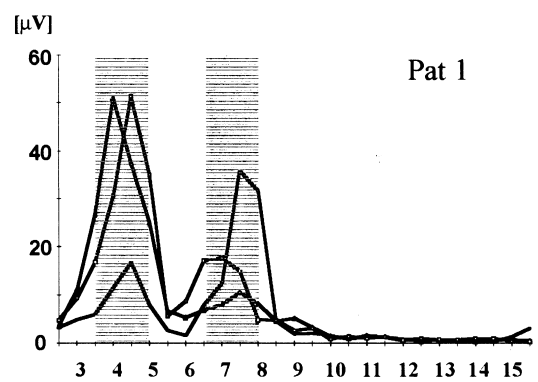

c)

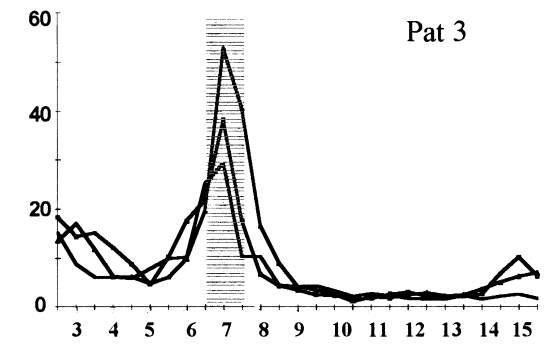

e)

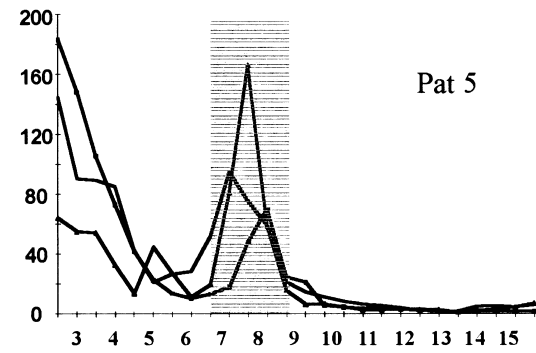

g)

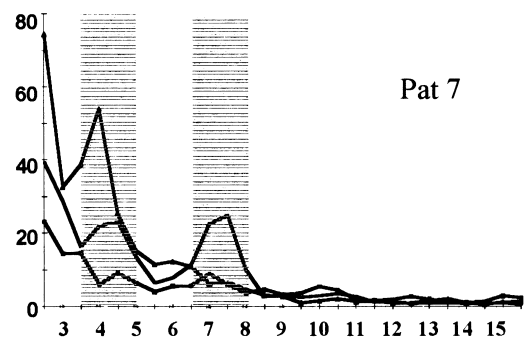

i)

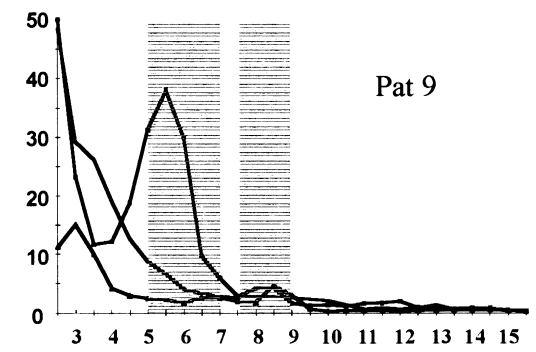

b)

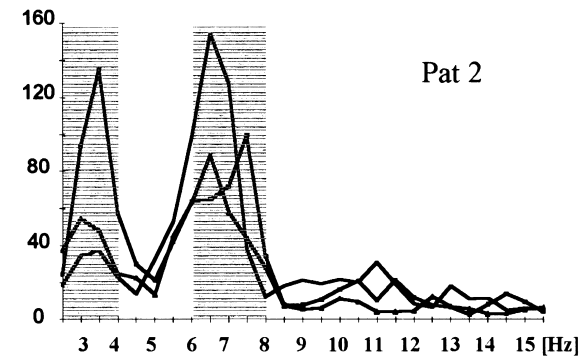

d)

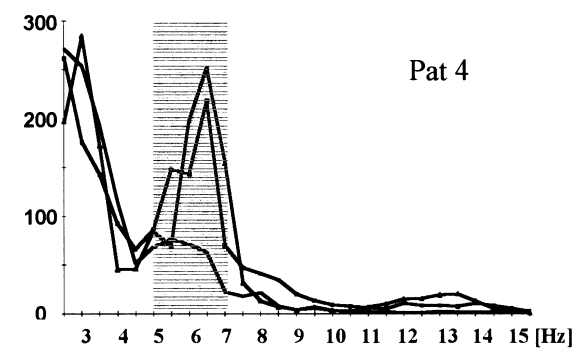

f)

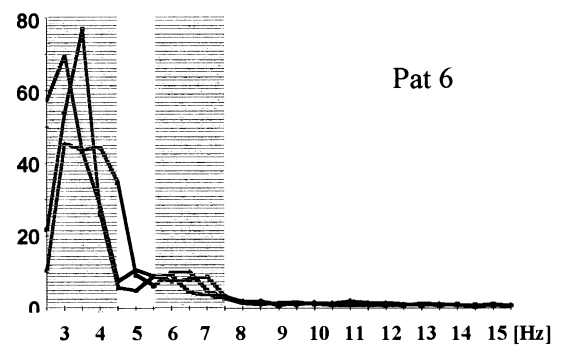

h)
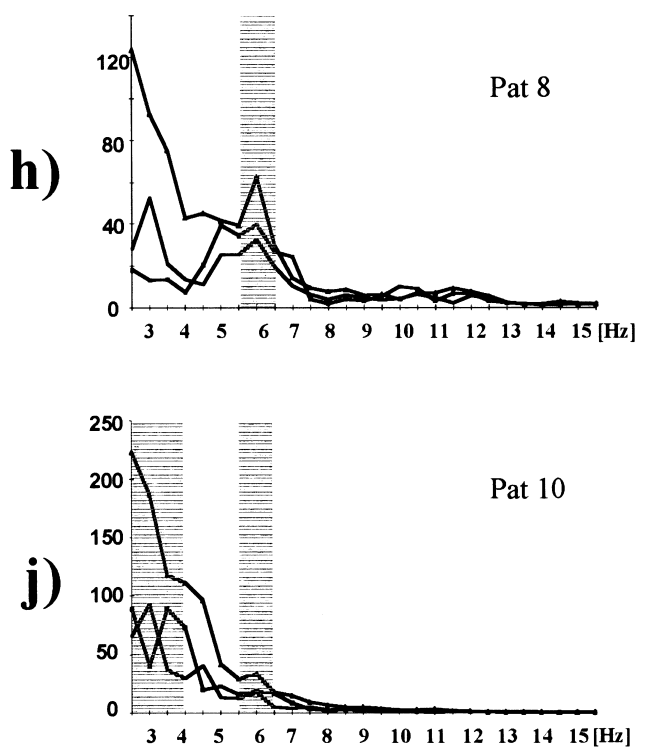

Fig. 2. Determination of the ictally dominant frequency band. For all 10 patients (a-j) and for all evaluated seizures (three per patient) the full scalp spectrum as determined by the FFT-Approximation as averaged over the $14 \mathrm{~s} \mathrm{period} \mathrm{following} \mathrm{the} \mathrm{electroencephalographic} \mathrm{seizure} \mathrm{onset} \mathrm{is} \mathrm{shown} \mathrm{from} 2.5$ to $15.5 \mathrm{~Hz}$. The global field power peaks for each seizure determined the ictally dominant frequencies in the respective seizure (i.e. in patient 5 (e), the dominant frequency was determined at 7.0,7.5, and $8.0 \mathrm{~Hz}$ ). The area indicated in gray represents the consistent (between three seizures) ictally dominant frequency band $( \pm 0.5 \mathrm{~Hz}$ around the power peak) which was subsequently analyzed. In 6 of 10 patients two consistent and dominant frequency bands could be calculated (see (a), patient 1 ). 
a)

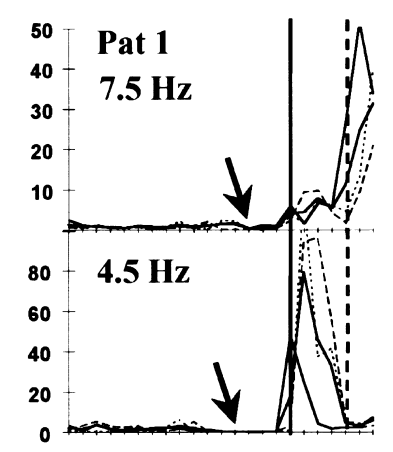

c)

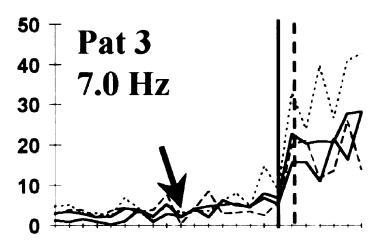

e)

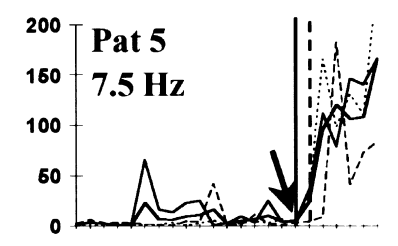

g)

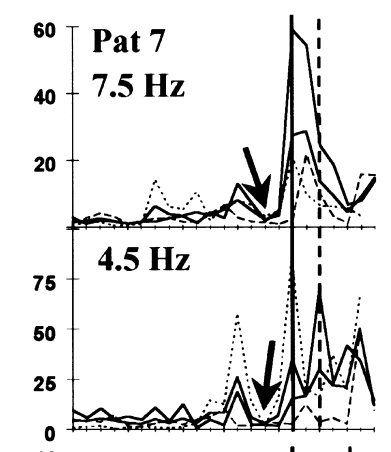

i)

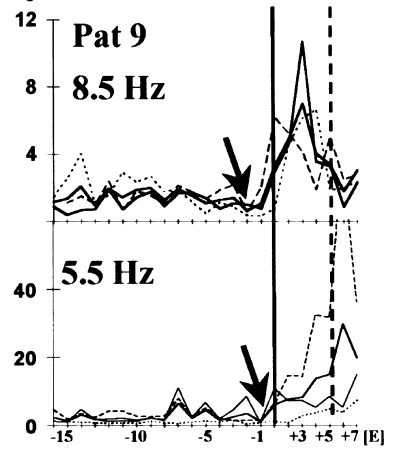

b)

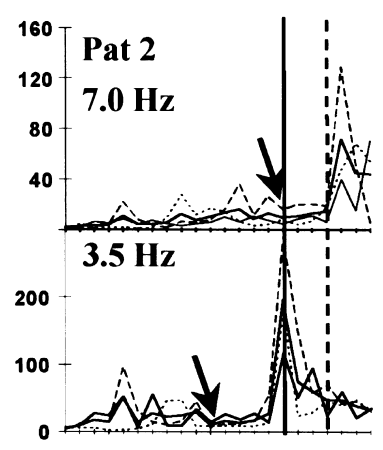

d)

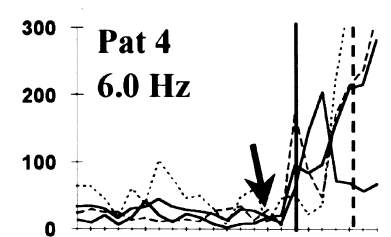

f)

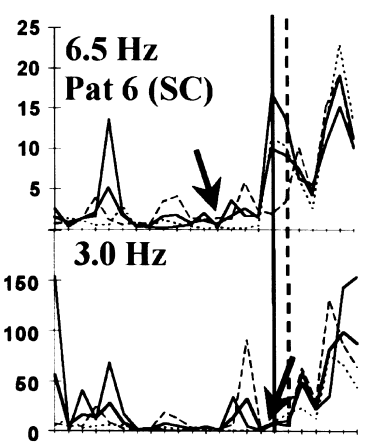

Pat 8

h)

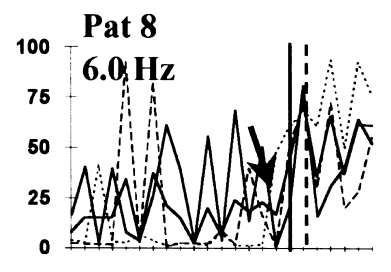

j)

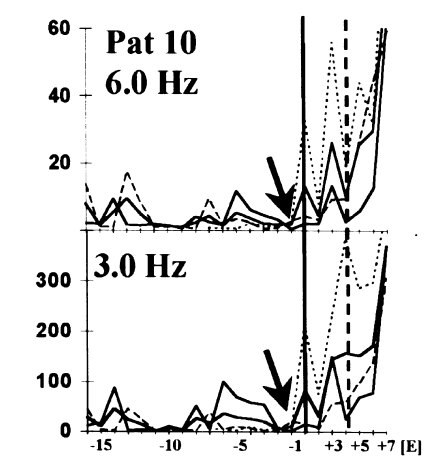

Fig. 3. The rise of the consistent and dominant frequency starts before the visual EEG onset. The temporal evolution of the global field power of the ictally dominant frequency bands is shown in all 10 patients $(\mathrm{a}-\mathrm{j})$. The temporal dynamics are shown for the whole evaluated period of $46 \mathrm{~s}$. The vertical line indicates the ESO and the dashed vertical line the RSO as in Fig. 1. Thus, in all patients, $14 \mathrm{~s}$ or seven $2 \mathrm{~s}$ time epochs (E) are shown after the electroencephalographic seizure onset (E1-E7, see $x$-axis in $(\mathrm{i}, \mathrm{j}))$ and $32 \mathrm{~s}$ or $16 \mathrm{E}$ are shown prior to the ESO (E-16-E-1). The dominant frequency of each evaluated seizure (three thin lines) over time are indicated for each patient. The fourth line (thick line) indicates the mean of the temporal evolution of the ictally dominant frequency. The arrow indicates its onset. 
Table 2

For each patient the ictally dominant frequency band $>5 \mathrm{~Hz}$ (given in bold) and the second frequency band (if present), the epoch (E) of their initial rise and their dominant epoch as well as the duration of its increase in strength are indicated ${ }^{\mathrm{a}}$

\begin{tabular}{|c|c|c|c|c|c|}
\hline Patient & $\begin{array}{l}\text { Ictal frequency } \\
(\mathrm{Hz})\end{array}$ & $\begin{array}{l}\text { Initial rise } \\
\text { (E) }\end{array}$ & $\begin{array}{l}\text { Dominant epoch } \\
\text { (E) }\end{array}$ & $\begin{array}{l}\text { RSO } \\
\text { (E) }\end{array}$ & $\begin{array}{l}\text { Duration of } \\
\text { GFP-increase (s) }\end{array}$ \\
\hline \multirow[t]{2}{*}{1} & 4.5 & -4 & 2 & 5 & 10 \\
\hline & 7.5 & -3 & 7 & 5 & 18 \\
\hline \multirow[t]{2}{*}{2} & 3.5 & -5 & 1 & 4 & 10 \\
\hline & 7 & 1 & 5 & 4 & 8 \\
\hline 3 & 7 & -7 & 7 & 2 & 26 \\
\hline 4 & 6 & -2 & 7 & 5 & 16 \\
\hline 5 & 7.5 & 1 & 7 & 2 & 12 \\
\hline \multirow[t]{2}{*}{6} & 3 & -1 & 6 & 2 & 12 \\
\hline & 6.5 & -4 & 6 & 2 & 18 \\
\hline \multirow[t]{2}{*}{7} & 4.5 & -2 & 6 & 3 & 14 \\
\hline & 7.5 & -2 & 2 & 3 & 6 \\
\hline 8 & 6 & -1 & 2 & 1 & 4 \\
\hline \multirow[t]{2}{*}{9} & 5.5 & -1 & 6 & 5 & 12 \\
\hline & 8.5 & -2 & 3 & 5 & 8 \\
\hline \multirow[t]{2}{*}{10} & 3 & -1 & 7 & 4 & 14 \\
\hline & 6 & -1 & 7 & 4 & 14 \\
\hline
\end{tabular}

E7. In patient 6 both frequencies $(3.0$ and $6.5 \mathrm{~Hz})$ peaked in E6 but the $6.5 \mathrm{~Hz}$ frequency increased its power already during E-4, the lower mean frequency during E-1. In the remaining patients only one dominant frequency was detected. In patient $37.0 \mathrm{~Hz}$ (Fig. 2c), in patient $46.0 \mathrm{~Hz}$ (Fig. 2d), in patient $57.5 \mathrm{~Hz}$ (Fig. 2e) and in patient $86.0 \mathrm{~Hz}$ (Fig. 2h). In patient 3 the dominant frequency increased its power from E-7 to E7 (Fig. 3c), in patient 4 from E-2 to E7 (Fig. 3d), in patient 5 from E1 to E7 (Fig. 3e) and in patient 8 the initial rise was found in E-1 and its DE in E2 (Fig. 3h). Even though a dominant ictal frequency could be clearly determined in patient 8 , the temporal evolution of this frequency band was found to show large variability within and across the three evaluated seizures (Fig. 3h). This pattern was clearly different if compared with the other patients, however if the mean of the frequency strength over time is calculated its increase prior to the ESO and the DE can be appreciated. Inspection of the EEG revealed high amplitude eye movement artifacts.

In summary, for each patient the duration from the initial increase to its maximal strength could be determined for the ictally dominant frequency. Over all patients, the mean time epoch with the initial rise was found in E-2. The dominant frequency was thus found $4.0 \mathrm{~s}$ prior to ESO and $9.0 \mathrm{~s}$ before the RSO. The DE was found on average in E6. The mean duration of the GFP-increase was $13.0 \mathrm{~s}$. In 6 of 10 patients a second dominant frequency which was smaller than $5 \mathrm{~Hz}$ was found. It started increasing its strength during E-3 (4.6 s prior to the ESO) and had its DE in E5. The mean duration of increase in strength was slightly shorter and started about $1 \mathrm{~s}$ earlier.

\subsection{Three-dimensional localization of seizure onset}

The spatial information contained in the ictally dominant frequency in the dominant time epoch was used to localize the epileptic activity with a linear inverse solution algorithm. The results of this analysis are shown in Fig. $4 a-j$ for all patients. The lateralization and localization of the mean dominant frequency during the period with its maximal strength corresponds for 9 of 10 patients well with the localization proposed by non-invasive methods such as EEG, MRI, PET and SPECT and with the operated brain region. The linear inverse solutions showed a very consistent localization on the lower slices in an anterior temporal or lateral temporal position (Fig. 3a-i). However more exact methods which use individual realistic head shapes, realistic head models and more complex linear inverse solutions will be necessary to distinguish between the ictal implication of lateral and mesial temporal structures (Fuchs et al., 1999; Michel et al., 1999). In patient 10 the EEG was characterized by strong eye movement artifacts which strongly influenced the localization procedure for both dominant frequencies (3.0 and $6.0 \mathrm{~Hz}$, see Fig. 4j).

\section{Discussion}

In a selected group of patients suffering from temporal lobe epilepsy in whom a mesiotemporal seizure origin can be assumed on the basis of EEG and MRI signs we analyzed the electric fields recorded by multichannel EEG in order to determine the time of onset and the further development of ictally dominant frequencies over time. By calculating the GFP of frequency-transformed phase corrected voltage maps, this method allowed to identify ictally dominant frequencies of $>5 \mathrm{~Hz}$ which started prior to initial EEG signs and incremented during the further evolution of the seizure in all patients. The ictally dominant frequencies found by the present approach thus confirms the importance 
a) Pat 1

b) Pat 2

c) Pat 3

d) Pat 4

e) Pat 5

f) Pat 6

g) Pat 7

h) Pat 8

i) Pat 9

j) Pat 10
$7.5 \mathrm{~Hz}$
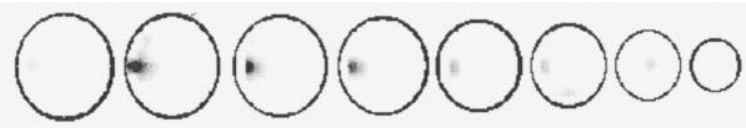

$7 \mathrm{~Hz}$

$7 \mathrm{~Hz}$

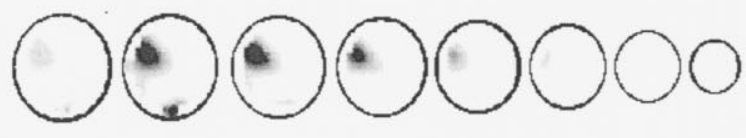

0

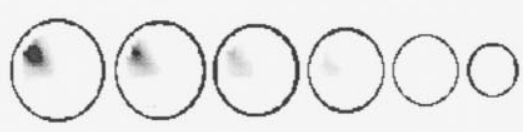

$6 \mathrm{~Hz}$
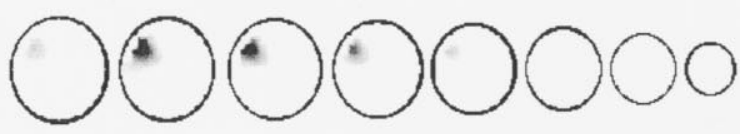

$7.5 \mathrm{~Hz}$

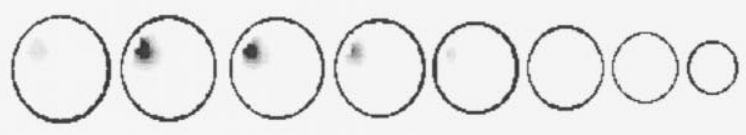

$6.5 \mathrm{~Hz}$
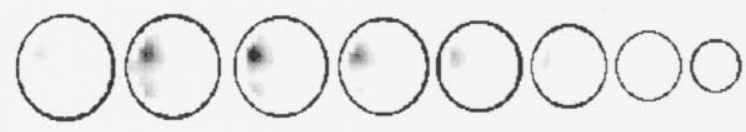

$7.5 \mathrm{~Hz}$

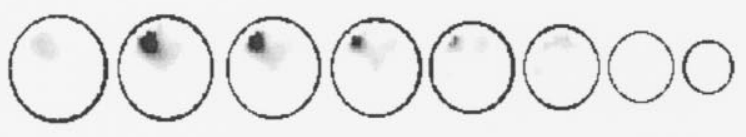

$6 \mathrm{~Hz}$

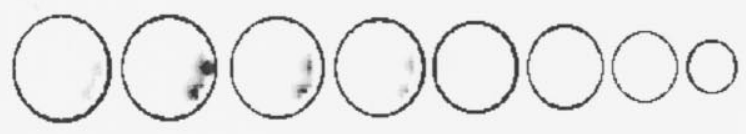

$8.5 \mathrm{~Hz}$

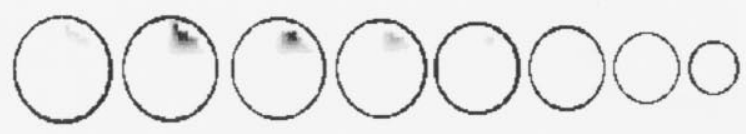

$6.5 \mathrm{~Hz}$
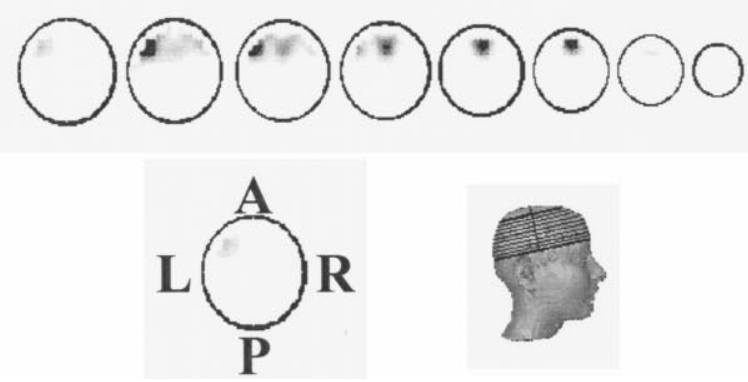

Fig. 4. 3D localization of the ictally dominant frequency band $(>5 \mathrm{~Hz})$ during the dominant epoch is shown for all patients in the same order as in Figs. 2 and 3. A distributed linear inverse solution was applied to the frequency domain potential maps. The figure in the lower right corner indicates the vertical position of the solution slices through the head: lower slices are shown for each patient on the left, higher slices (at the top of the head) on the right. L, left; R, right; A, anterior; P, posterior for each shown slice. Note that in 9 out of 10 patients, the ictally dominant frequency during the dominant epoch is lateralized and localized to the brain region which has been operated. In patient 10, a bifrontal activation was obtained for the dominant frequency.

of ictal frequency patterns $>5 \mathrm{~Hz}$ - as found by visual EEG analysis - in mesial temporal lobe epilepsy (Risinger et al., 1989; Ebersole and Pacia, 1996; Pacia and Ebersole, 1997).
Furthermore, Risinger et al. (1989) argued that the appearance of this pattern during early and later ictal periods $(>30$ $\mathrm{s}$ following the seizure onset) is characteristic of mesial 
temporal lobe onset, whereas Ebersole and Pacia (1996) only found a good specificity for a mesiotemporal onset if the pattern appeared during the first five seconds of the seizure. The early detection of the ictally dominant frequency in all patients by the use of EEG mapping techniques in the frequency domain suggests that especially the early appearance of this pattern immediately after the ESO is a strong indicator of a mesial temporal onset as suggested by Ebersole and Pacia (1996). Furthermore we did not observe initial rises of ictally dominant frequencies during later epochs of the seizure (i.e. E5-E7). However, since we did only analyze early time periods during seizures (up to 14 s following the ESO), we cannot exclude that the 'delayed focal onset' ( $>30 \mathrm{~s}$ after ESO) as proposed by Risinger et al. (1989) also predicts mesial temporal lobe epilepsy.

The range of ictal frequencies in our patient group was somewhat larger than in previously reported studies and in 6 of 10 patients a second mean frequency peak at $4.0 \mathrm{~Hz}$ was determined. Whereas Risinger et al. (1989) do not mention the appearance of frequencies $<5 \mathrm{~Hz}$ in mesial temporal lobe epilepsy, these frequencies have been found in $16 \%$ of patients with mesiotemporal onset as determined by intracranial recordings (Pacia and Ebersole, 1997). Minor spectral changes of ictal frequencies (i.e. from 5 to $7 \mathrm{~Hz}$ ) can also be appreciated in the figures of the exemplary seizures of mesiotemporal origin in the paper by Ebersole and Pacia (1996). Finally, Lantz et al. (1999) have also observed in some of their patients that during different time periods following the ESO different frequencies become dominant. Even if our analysis confirms the importance of ictal frequencies $>5 \mathrm{~Hz}$ in mesiotemporal lobe epilepsy since they could be detected in all patients, we note that in 4 of 6 patients in whom two dominant frequencies were determined the lower frequencies from 3.0 to $5.5 \mathrm{~Hz}$ appeared earlier than or at the same time as the higher 'typical mesiotemporal' frequency patterns. This quantitative finding confirms qualitative EEG analysis (Pacia and Ebersole, 1997) and might suggest an early ictal implication of lateral temporal structures in some cases of mesial temporal lobe epilepsy. Future quantitative EEG-studies which investigate seizures arising in the lateral temporal lobe will further clarify the frequential differentiation between lateral and mesial temporal seizures. Another important finding in our study is the onset of the dominant frequencies $>5 \mathrm{~Hz}$ prior to the ESO and its consistent increase in strength over a period of $13 \mathrm{~s}$. The onset of the ictally dominant frequencies was found prior to non-focal EEG signs (ESO) and focal EEG signs (RSO) in a time period in which visual EEG analysis did not reveal focal epileptogenic activity. The initial rise of the ictally dominant frequencies was determined as early as $16 \mathrm{~s}$ prior to the onset of focal rhythmic activity (see patient 3, Fig. 3c). This period prior to focal RSO in surface EEG (Risinger et al., 1989; Ebersole and Pacia, 1996) has recently been investigated by Pacia and Ebersole (1997) in simultaneous depth and surface (8-20 electrodes) recordings. In their investigation this period lasted from $10 \mathrm{~s}$ to $>1 \mathrm{~min}$ and 'showed either no change or a diffuse disruption of the background' activity in surface EEG recordings. During this silent EEG period, simultaneous temporal depth electrodes recorded focal ictal rhythms of $>13 \mathrm{~Hz}$ which have been described by Spencer et al. (1992) as the typical hippocampal seizure onset. The onset of focal rhythmic activity in surface EEG recordings (RSO in our study) characterizes the next ictal phase and shows a spectral pattern of $>5 \mathrm{~Hz}$ which is supposed to arise from synchronously active lateral and mesial temporal structures (Pacia and Ebersole, 1997). Three lines of evidence in our results suggest that the combination of multichannel EEG recordings with topographic mapping methods in the frequency domain might allow to detect ictal rhythms before the pathological seizure activity has encompassed mesial as well as lateral temporal structures: (1) the appearance of the ictally dominant frequency $9.0 \mathrm{~s}$ prior to rhythmic seizure onset with its long continuous duration; and (2) the onset of this frequency even prior (4.0 s) to non-focal EEG signs (ESO) in almost all evaluated seizures. The present approach allows to follow the ictally dominant frequencies back in time into a period when the mesial ictal activity - as found by simultaneous surface and depth recordings - has not yet spread to the lateral temporal neocortex (Pacia and Ebersole, 1997). Although this ictal pattern can only be cautiously assumed in our data - since our patients were operated without previous intracranial seizure monitoring - the precise selection of our patients, the excellent postoperative seizure outcome and the neuropathological proof of hippocampal sclerosis in most cases render it highly probable that in all cases the seizure originated in the mesiotemporal lobe. Furthermore, (3) Michel et al. (1999) were recently able to detect focal ictal epileptic rhythms restricted to the hippocampus by means of surface electrodes in using the technique used here in a patient with simultaneous depth and surface recordings. Our results therefore suggest that the calculation of the global field power of phase corrected voltage maps in the frequency domain can be reliably used to detect - early in the development of a seizure - focal pathological activity arising in deep structures such as the mesial temporal lobe. It is furthermore suggested that the observed increase of the power of the ictally dominant frequency over time might be related to the amount of epileptogenic tissue involved in the generation of the ictal discharges, starting in the mesial temporal lobe and propagating later, following the ESO as proposed by Pacia and Ebersole (1997) to neocortical structures.

Our data further demonstrate in 9 of 10 cases that the surface distribution of the EEG in the ictal frequency contains spatial information that can be used to correctly lateralize and localize the ictal focus, by using the combination of the FFT-Approximation and a linear inverse solution. This localization is concordant with the results obtained with other non-invasive evaluation methods such as EEG, MRI, PET and SPECT and shows that the observed 
spectral changes during the early evolution of the evaluated seizures are related to the epileptogenic sources in the temporal lobe whose resection rendered all patients seizure-free. This is especially interesting since visual EEG analysis does not reveal any focal EEG changes at that time (Ebersole and Pacia, 1996). However this localization procedure alone does not allow to decide if the primary epileptic focus is in mesial or lateral structures, or already in both areas (Lantz et al., 1997). Future EEG mapping studies in patients with neocortical temporal lobe epilepsy might show a different localization of the epileptic focus and a different temporal evolution of ictal rhythms during seizures in comparison to seizures arising in the mesial temporal lobe. These findings are clinically important since they might help to non-invasively identify patients suffering from seizures arising in the temporal neocortex, because they have less favorable outcomes with standard partial anterior temporal lobectomy. As discussed elsewhere the combination of multiple linear and non-linear inverse solutions as well as realistic head models seems mandatory in order to use a localization method to its full potential (Ebersole, 1999; Fuchs et al., 1999; Michel et al., 1999).

In conclusion, we believe that this methodological approach can accurately and reliably define a patient-specific ictal frequency pattern which is generated initially by a small ictal region followed by discharges situated in a large electrical field later in the seizure. Since surface ictal recordings are considered by many to provide the best non-invasive indicator of the epileptogenic zone, we think that easy comparable criteria and maximized interobserver reliability are strongly needed in order to further improve the utilization of surface EEG recordings to their full potential. The findings of the present study suggest that a combination of spatial mapping techniques, full-scalp spectral analysis and linear inverse solutions might be a useful tool during the presurgical noninvasive evaluation phase.

\section{Acknowledgements}

Supported by the Swiss National Science Foundation grant Nos. 31-57112.99 and 31-52933.97.

\section{References}

Alcaron G, Binnie CD, Elwes RDC, Polkey CE. Power spectrum and intracranial EEG patterns at seizure onset in partial epilepsy. Electroenceph clin Neurophysiol 1995;94:326-337.

Blanke O, Seeck M, Lantz G, Landis T, Michel CM. Localisation of the site of seizure onset using frequency-domain source localisation. Epilepsia 1997;38(Suppl 3):155.

Blume WT, Young GB, Lemieux JF. Clinical and electroencephalographic correlates of multiple independent spike foci pattern in children. Ann Neurol 1984;4:541-547.

Dierks T, Engelhardt W, Maurer K. Equivalent dipoles of FFT data visualize drug interactions at benzodiazepine receptors. Electroenceph clin Neurophysiol 1993;86:231-237.

Ebersole JS. Last word. J Clin Neurophysiol 1999;16:297-302.

Ebersole JS, Pacia SV. Localization of temporal lobe foci by ictal EEG patterns. Epilepsia 1996;37:386-399.

Engel J. Intracerebral recordings: organization of the human epileptogenic region. J Clin Neurophysiol 1993;10(1):90-98.

Fuchs M, Wagner M, Kohler T, Wischmann HA. Linear and nonlinear current density reconstructions. J Clin Neurophysiol 1999;16:267-295.

Geiger LR, Harner RN. EEG patterns at the time of focal seizure onset. Arch Neurol 1978;35:276-286.

Gotman J, Levtova V, Farine B. Graphic representation of the EEG during epileptic seizures. Electroenceph clin Neurophysiol 1993;87:206-214.

Grave de Peralta Menendez R. Linear inverse solutions. Ph.D. Thesis, Geneva, 1999.

Grave de Peralta Menendez R, Gonzalez Andino SL. A critical analysis of linear inverse solutions. IEEE Trans Biomed Engineer 1998;45:440448.

Lantz G, Michel CM, Pasqual-Marqui R, Spinelli L, Seeck M, Seri S, Landis T, Rosen I. Extracranial localization of intracranial epileptiform activity using LORETA (low resolution electromagnetic tomography). Electroenceph clin Neurophysiol 1997;102:414-422.

Lantz G, Michel CM, Seeck M, Blanke O, Landis T, Rosen I. Frequency domain EEG source localization of ictal epileptiform activity in patients with partial complex epilepsy of temporal lobe origin. Electroenceph clin Neurophysiol 1999;110:176-184.

Lehmann D, Michel CM. Intracerebral dipole source localization for FFT power maps. Electroenceph clin Neurophysiol 1990;76:271-276.

Lehmann D, Henggeler B, Koukkou M, Michel CM. Source localization of brain electric field frequency bands during conscious, spontaneous visual imagery and abstract thoughts. Cogn Brain Res 1993;1:203210.

Michel CM, Lehmann D, Henggeler B, Brandeis D. Localization of the sources of EEG delta, theta, alpha and beta frequency bands using FFT dipole approximation. Electroenceph clin Neurophysiol 1992;82:38-44.

Michel CM, Pascual-Marqui RD, Strik WK, Koenig T, Lehmann D. Frequency domain source localization shows state dependent diazepam effects in 47-channel EEG. J Neural Transm Gen Sect 1995;99:157171.

Michel CM, Grave de Peralta R, Lantz G, Gonzalez Andino S, Spinelli L, Blanke O, Landis T, Seeck M. Spatio-temporal analysis and distributed source estimation in presurgical epilepsy evaluation. J Clin Neurophysiol 1999;16:239-266.

Pacia SV, Ebersole JS. Intracranial EEG substrates of scalp ictal patterns from temporal lobe foci. Epilepsia 1997;38:642-654.

Quesney LF, Risinger MW, Shewmon DA. Extracranial EEG evaluation. In: Engel J, editor. Surgical treatment of the epilepsies, New York: Raven Press, 1993. pp. 173-195.

Risinger NW, Engel J, Van Ness PC, Henry TR, Crandall PH. Ictal localization of temporal lobe seizures with scalp/sphenoidal recordings. Neurology 1989;39:1288-1293.

Spencer SS, Spencer DD, Williamson PD, Mattson RH. Combined depth and subdural electrode investigation in uncontrolled epilepsy. Neurology 1990;40:74-79.

Spencer SS, Guimares P, Katz A, Kim J, Spencer DD. Morphological patterns of seizures recorded intracranially. Epilepsia 1992;33:537-545.

Spinelli L, Pascual-Marqui R, Grave de Peralta-Menendez R, Michel CM. Effect of the number and the configuration of electrodes on the distributed source models. Brain Topogr 1997;10:88. 